Pacific Journal of Mathematics

MAXIMAL CONVEX FILTERS IN A LOCALLY CONVEX 


\title{
MAXIMAL CONVEX FILTERS IN A LOCALLY CONVEX SPACE
}

\author{
F. J. WAGNER
}

Let $E[\mathscr{T}]$ be a locally convex space, $\mathfrak{B}$ a saturated covering of $E$ by bounded sets, and $E^{\prime}$ the topological dual of $E[\mathscr{T}]$. Let $\mathscr{T}_{\mathfrak{B}}$ be the topology on $E^{\prime}$ of uniform convergence on sets of $\mathfrak{B}$ and $E^{\prime \prime}$ the topological dual of $E^{\prime}$ [ $\mathscr{T}_{\mathfrak{B}}$ ]. We assume $E^{\prime \prime}$ has the natural topology $\mathscr{T}_{n}$ - that of uniform convergence on the equicontinuous sets of $E^{\prime}$.

This article includes the following : (1) an intrinsic characterization for a bounded convex set $B$ of $E$ of the closure $\bar{B}$ of $B$ in $E^{\prime \prime}$; (2) an intrinsic characterization of the closure $\bar{E}$ of $E$ in $E^{\prime \prime}$; and (3) necessary and sufficient conditions that $\bar{E}$ be $E^{\prime \prime}$.

The spaces $\beta$. Let $\mathfrak{M}$ be the class of all closed convex neighborhoods ${ }^{1}$ of 0 in $E[\mathscr{T}]$, and $B \in \mathfrak{B}$. A filter $\mathfrak{F}$ on $B$ is called a convex filter if, for every $F \in \mathfrak{F}$, there exist $M, N \in \mathfrak{M}$ and $\chi \in E$ such that $\stackrel{\circ}{\mathrm{D}} \supset N, F \supset(M+\chi) \cap B$, and $(N+\chi) \cap B \in \mathfrak{F}$. Clearly if $\mathfrak{F}$ and $\mathbb{S}$ are two convex filters on $B$, such that every set of $\widetilde{F}$ meets every set of $\mathbb{B}$, then the least upper bound filter of $\mathfrak{F}$ and $\mathbb{B}$ on $B$ is also convex. Furthermore :

LEMma 1. For $M, N \in \mathfrak{M}$, if $\stackrel{\circ}{M} \supset N$, then there exists $K \in \mathfrak{M}$ such that $\stackrel{\circ}{M} \supset$ $\supset \stackrel{\circ}{K} \supset N$.

Proof. If $p$ and $q$ are the distance functions of $M$ and $N$, then $1 / 2(p+q)$ is the distance function of such a $K$.

THEOREM 1. A convex filter $\mathfrak{F}$ on $B$ is a maximal convex filter on $B$ if and only if, for every two closed convex bodies $K$ and $L$ of $E$ such that $\stackrel{\circ}{K} \supset L$, either $K \cap B \in \mathfrak{F}$ or $B \backslash L \in \mathfrak{F}$.

Proof. Assume $\mathfrak{F}$ is maximal and let $K$ and $L$ be as above, and let $B \backslash L \notin \mathfrak{F}$. Let $x \in \stackrel{\circ}{L}$ and define a sequence $\left\{M_{n}\right\}$ in $\mathfrak{M}$ so that

$$
\stackrel{\circ}{K}-x \supset M_{1} \supset \stackrel{\circ}{M}_{1} \supset L-x \text { and } \stackrel{\circ}{M}_{n} \supset M_{n+1} \supset \stackrel{\circ}{M}_{n+1} \supset L-x \quad(n \geqq 1) .
$$

Then the filter \&s on $B$ with base $\left\{\left(M_{n}+x\right) \cap B \mid n=1,2,3, \cdots\right\}$ is

Roceived July 8, 1964 and in revised form January 11, 1965. Supported by Nullonal Science Foundation grant NSF G-24865.

- The notation and definitions are principally those of Gottfried Köthe, Topolowiwhe I.Ineure Räume I, Springer-Verlag, Berlin, 1960. 
convex and $K \cap B \in \mathbb{B} \subset \mathfrak{F}$.

Conversely let $\mathfrak{F}$ and $\mathbb{S}$ be two convex filters on $B$ such that $\mathfrak{F}$ is strictly weaker than $\mathbb{S}$. Let $G \in \mathbb{E}, M, N \in \mathfrak{M}$, and $x \in E$ such that $G \notin \mathfrak{F}, \stackrel{\circ}{M} \supset N, G \supset(M+x) \cap B$, and $(N+x) \cap B \in \mathbb{F}$. Then neither $(M+x) \cap B$ nor $B \backslash(L+x) \in \mathfrak{F}$.

REMARKS 1. For every $x \in B, \mathfrak{B}_{B}(x)=\{V \cap B \subset B \mid V$ a neighborhood of $x$ in $E$ \} is a maximal convex filter on $B$.

2. For a maximal convex filter $\mathfrak{F}$ on $B$, there is $x \in B$ such that $\mathfrak{F}=\mathfrak{V}_{B}(x)$ if and only if $\mathfrak{F}$ has nonempty intersection.

Lemma 2. Every maximal convex filter on $B$ is a weak Cauchy filter.

Proof. Let $\mathfrak{\&}$ be a maximal convex filter on $B$, $u \in E^{\prime}, M=\{x \in E|| u x \mid \leqq 1 / 2\} \quad$ and $\quad N=\{x \in E|| u x \mid \leqq 1 / 4\}$.

Then $M, N \in \mathfrak{M}$ and $\stackrel{\circ}{M} \supset N$. Since $B$ is weakly precompact, there exist $x_{1}, x_{2}, \cdots, x_{n} \in E$ such that $\bigcup_{i=1}^{n}\left(N+x_{i}\right) \supset B$, and so $\left(M+x_{i}\right) \cap B \in \mathfrak{F}$ for some $1 \leqq i \leqq n$. For $x, y \in\left(M+x_{i}\right) \cap B$, we have $|u x-u y| \leqq 1$.

For a maximal convex filter $\mathfrak{F}$ on $B$ and $u \in E^{\prime}$, let $\mathfrak{F}(u)$ denote the limit of the restriction of $u$ to $B$ according to the filter $\mathfrak{F}$.

LEMMA 3. For every maximal convex filter $\mathfrak{F}$ on $B$, the mapping $u \rightarrow \mathfrak{F}(u)$ on $E^{\prime}$ is linear and $\mathscr{T}_{\mathfrak{B}}$ continuous.

Proof. Linearity is easily proved. Also let $V$ be the polar set of the absolutely convex hull of $2 B, u \in V$, and $F \in \mathfrak{F}$ such that $|u x-\mathfrak{F}(u)| \leqq 1 / 2$ for every $x \in F$. Then, for such an $x$, we have $|\mathfrak{\mho}(u)| \leqq|\mathfrak{F}(u)-u x|+|u x| \leqq 1$.

We shall denote by $\beta=\beta_{B}$ the set of all maximal convex filters on B. By Lemma 3 there is a mapping $\pi_{B}$ from $\beta_{B}$ into $E^{\prime \prime}$ such that $\pi_{B}(\mathfrak{F})(u)=\mathfrak{F}(u)$ for every $\mathfrak{F} \in \beta_{B}$ and $u \in E^{\prime}$.

THEOREM 2. If either $\mathscr{T}$ is the weak topology or $B$ is convex, then $\pi_{B}$ is a one-to-one mapping of $\beta_{B}$ onto the $\mathscr{T}_{n}$-closure $\bar{B}$ of $B$ in $E^{\prime \prime}$.

Proof. For $\mathfrak{F} \in \beta_{B}, \pi_{B}(\mathfrak{F})$ is in the weak closure of $B$ in $E^{\prime \prime}$. For 
given $u_{1}, \cdots, u_{n} \in E^{\prime}$ and $\varepsilon>0$, let $F_{1}, \cdots, F_{n} \in \mathfrak{F}$ such that $\left|u_{i} x-\mathfrak{F}\left(u_{i}\right)\right| \leqq \varepsilon(1 \leqq i \leqq n)$ and $x \in \bigcap_{i=1}^{n} F_{i}$. Then $\left|\mathfrak{F}\left(u_{i}\right)-u_{i} x\right| \leqq \varepsilon$, $(1 \leqq i \leqq n)$.

Also, if $B$ is convex, $\pi_{B}(\mathfrak{F})$ is in the $\mathscr{T}_{n}$-closure $\bar{B}$ of $B$ in $E^{\prime \prime}$. Suppose the contrary. Then there is a continuous real linear functional $w$ on $E^{\prime \prime}$ and a real number $r$ such that $w\left(\pi_{B}(\mathfrak{F})\right)<r$ and $w z>r$ for every $z \in \bar{B}$.

Assume first that $E$ is a real vector space. Let $u$ be the restriction of $w$ to $E^{\prime}$, so $u \in E$. Let $F \in \mathfrak{F}$ such that $|u x-\mathfrak{F}(u)|<r-w\left(\pi_{B}(\mathfrak{F})\right)$ for every $x \in F$. Then, for such an $x$, we have $w x=u x-\mathfrak{F}(u)+$ $\widetilde{\mho}(u)<r$. But $x \in B$.

Now let $E$ be a complex vector space. Then there is a complex linear functional $v$ on $E^{\prime \prime}$ such that $w=\Re v$. Let $u$ be the restriction of $v$ to $E$ and $F \in \mathfrak{F}$ such that $|u x-\mathfrak{F}(u)| \leqq r-w\left(\pi_{B}(\mathfrak{F})\right)$ for every $x \in F$. Then for such an $x$ we have $w x=\Re(v x)=\Re(u x-\mathfrak{F}(u))+$ $\Re(\mathfrak{F}(u))<r$. Again, we have a contradiction.

Thus $\pi_{B}\left(\beta_{B}\right) \subset \bar{B}$ if $\mathscr{T}$ is the weak topology or $B$ is convex.

On the other hand, if $z \in \bar{B}$, then:

$$
\mathfrak{B}_{B}(z)=\left\{V \cap B \subset B \mid V \text { a neighborhood of } z \text { in } E^{\prime \prime}\left[\mathscr{T}_{n}\right]\right\} \in \beta_{B}
$$

and $\pi_{B}\left(\mathfrak{V}_{B}(z)\right)=z$. Let $V$ be a neighborhood of $z$ in $E^{\prime \prime}\left[\mathscr{T}_{n}\right]$, and let $U$ and $W$ be closed convex neighborhoods of 0 in $E^{\prime \prime}\left[\mathscr{T}_{n}\right]$ such that $\stackrel{\circ}{U} \supset W$ and $U+U \subset V-z$. Let $\chi \in(U+z) \cap(-\stackrel{\circ}{W}+z) \cap B, \quad M=$

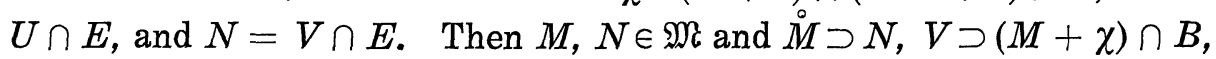
and $(N+\chi) \cap B=(W+\chi) \cap B \in \mathfrak{B}_{B}(z)$. Thus $\mathfrak{B}_{B}(z)$ is convex.

Let $K$ and $L$ be closed convex bodies of $E$ such that $\stackrel{\circ}{K} \supset L$. Let $x \in \stackrel{\circ}{L}, M=K-x$, and $N=L-x$. Either $z \in$ interior $M^{\circ \circ}+x-$ in which case $K \cap B=(M+x) \cap B=\left(M^{\circ \circ}+x\right) \cap B \in \mathfrak{B}_{B}(z)-$ or $z \notin N^{\circ \circ}+$ $x$ - in which case $E^{\prime \prime} \backslash\left(N^{\circ \circ}+x\right)$ is a neighborhood of $z$ in $E^{\prime \prime}$ and so $B \backslash L=\left[E^{\prime \prime} \backslash\left(N^{\circ \circ}+x\right)\right] \cap B \in \mathfrak{B}_{B}(z)$. Thus $\mathfrak{B}_{B}(z) \in \beta_{B}$.

Finally, let $u \in E^{\prime}, \varepsilon>0$, and $F \in \mathfrak{B}_{B}(z)$ such that $\left|u x-\mathfrak{B}_{B}(z)(u)\right| \leqq$ $\varepsilon / 2$ for every $x \in F$. Let $V=\left\{w \in E^{\prime \prime}|| w u-z u \mid \leqq \varepsilon / 2\right\}$. Then, for $x \in F \cap V$, we have $\left|\mathfrak{F}_{B}(z)(u)-z u\right| \leqq\left|\mathfrak{B}_{B}(z)(u)-u x\right|+|u x-z u| \leqq \varepsilon$. Therefore, $\pi_{B}\left(\mathfrak{B}_{B}(z)\right)(u)=z u$ for $u \in E^{\prime \prime}$, and so $\pi_{B}\left(\mathfrak{B}_{B}(z)\right)=z$.

REMARK. Thus $\pi_{B}\left(\mathfrak{B}_{B}(z)\right)=z$ for $z \in \bar{B}$ and $\mathfrak{F}=\mathfrak{B}_{B}\left(\pi_{B}(\mathfrak{F})\right)$ for $\mathfrak{F} \in \beta_{B}$.

COROLlaRY 1. If either $\mathscr{T}$ is the weak topology or $B$ is convex, then every maximal convex filter on $B$ is a $\mathscr{T}$-Cauchy filter.

(OROLlary 2. If either $\mathscr{T}$ is the weak topology or $B$ is convex, 
then for every $\mathfrak{F} \in \beta_{B}$ and $M \in \mathfrak{M}$, there exist $x \in B$ such that $(M+x) \cap B \in \mathfrak{F}$.

Proof. Let $F \in \mathfrak{F}$ such that $F^{\prime}-F \subset M$ and $x \in F$.

For $M \in \mathfrak{M}$ and $x \in B$ we define:

$$
\begin{aligned}
& \nu_{B}(M, x)=\left\{\mathfrak{F} \in \beta_{B} \mid(\stackrel{\circ}{M}+x) \cap B \in \mathfrak{F}\right\} \\
& \mu_{B}(M, x)=\left\{\mathfrak{F} \in \beta_{B} \mid \pi_{B}(\mathfrak{F}) \in \text { interior } M^{\circ \circ}+x\right\} .
\end{aligned}
$$

For $M, N \in \mathfrak{M}$ and $x, y \in B$, if $z \in(\stackrel{\circ}{M}+x) \cap(\stackrel{\circ}{N}+y) \cap B$ and $K=(M+x-z) \cap(N+y-z)$, then $\nu_{B}(M, x) \cap \nu_{B}(N, y)=\nu_{B}(K, z)$ and $\mu_{B}(M, x) \cap \mu_{B}(N, y)=\mu_{B}(K, z)$. Hence the class of all sets of the form $\nu_{B}(M, x)$ and the class of all sets of the form $\mu_{B}(M, x)$ (for $M \in \mathbb{M}$ and $x \in B$ ) form bases of topologies, called the $\nu$ - and $\mu$-topologies respectively, on $\beta_{B}$.

Theorem 3. If $\pi_{B}\left(\beta_{B}\right) \subset \bar{B}$ (in particular if either $\mathscr{T}$ is the weak topology or $B$ is convex), then $\nu$-and $\mu$-topologies coincide and $\pi_{B}$ is a homeomorphism of $\beta_{B}$ onto $\bar{B}$.

Proof. If $\pi_{B}\left(\beta_{B}\right) \subset \bar{B}$, then, for $M \in \mathfrak{M}$ and $x \in B$, we have $\mu_{B}(M, x) \subset \nu_{B}(M, x)$, and so the identity mapping of $\beta_{B}$ with the $\mu$ topology onto $\beta_{B}$ with the $\nu$-topology is continuous.

Also $\pi_{B}$ from $\beta_{B}$ with the $\nu$-topology onto $\bar{B}$ is continuous. Let $\mathfrak{F} \in \beta_{B}$ and $V$ a neightorhood of $\pi_{B}(\mathfrak{F})$ in $E^{\prime \prime}\left[\mathscr{T}_{n}\right]$. Let $U$ be a closed convex neighborhood of 0 in $E^{\prime \prime}$ such that $U+U \subset V-\pi_{B}(\mathfrak{F}), \quad M=$ $U \cap E$, and $x \in\left(\stackrel{\circ}{U}+\pi_{B}(\mathfrak{F})\right) \cap B$. Then $(\stackrel{\circ}{M}+x) \cap B \in \mathfrak{B}_{B}\left(\pi_{B}(\mathfrak{F})\right)=\mathfrak{F}$, and so $\mathfrak{F} \in \nu_{B}(M, x)$. Also if $\mathbb{S} \in \nu_{B}(M, x)$, there is a neighborhood $W$ of $\pi_{B}(\mathbb{S})$ such that $W \cap B=(M+x) \cap B=(U+x) \cap B$ so

$$
\pi_{B}(\mathbb{S}) \in \overline{W \cap B} \subset U+x \subset U+U+\pi_{B}(\mathfrak{F}) \subset V .
$$

Finally $\pi_{B}^{-1}$ from $\bar{B}$ onto $\beta_{B}$ with the $\mu$-topology is continuous by the definition of the sets $\mu$.

COROLLARY 1. If either $\mathscr{T}$ is the weak topology or $B$ is convex, then $B$ is closed in $E^{\prime \prime}\left[\mathscr{T}_{n}\right]$ if and only if every maximal convex filter on $B$ has nonempty intersection.

COROLLARY 2. $B$ is weakly compact if and only if every maximal weakly convex filter on $B$ has nonempty intersection.

2. The space $\eta$. Let $\mathfrak{A}$ denote the class of all convex sets of $\mathfrak{B}$ and $\alpha=\mathrm{U}_{B \in \mathfrak{X}} \beta_{B}$ the topological union of the spaces $\beta_{B}$. Let $\pi$ be 
the continuous function from $\alpha$ into $E^{\prime \prime}\left[\mathscr{T}_{n}\right]$ defined by $\pi(\mathfrak{F})=\pi_{B}(\mathfrak{F})$ if $\mathfrak{F} \in \beta_{B}$. For $A, B \in \mathfrak{N}$ such that $A \subset B$, define a mapping $g_{B A}$ from $\beta_{A}$ into $\beta_{B}$ by $g_{B A}(\mathfrak{F})=\mathfrak{B}_{B}\left(\pi_{A}(\mathfrak{F})\right.$ ) (for $\mathfrak{F} \in \beta_{A}$ ). Then $g_{B A}=\pi_{B}^{-1} \pi_{A}$ and consequently is a homeomorphism of $\beta_{A}$ into $\beta_{B}$. Also, if $A \subset B \subset C$, then $g_{O_{A}}=g_{O B} g_{B A}$.

Theorem 4. Let $A, B \in \mathfrak{A}$ such that $A \subset B$, and let $\mathfrak{F} \in \beta_{A}$ and (S) $\in \beta_{B}$. The following three conditions are equivalent;
(a) (S) $=g_{B A}(\mathfrak{F})$;
(b) $\pi(\mathfrak{\mho})=\pi(\mathbb{S})$;
(c) Every set of (B) contains a set of $\mathfrak{F}$.

Proof. $\mathfrak{F}=\mathfrak{B}_{A}\left(\pi_{B}(\mathfrak{F})\right), \quad \mathbb{S}=\mathfrak{B}_{B}\left(\pi_{B}(\mathbb{S})\right), \quad$ and $\quad g_{B A}(\mathfrak{F})=\mathfrak{B}_{B}\left(\pi_{B}(\mathfrak{F})\right)$. Hence (a) and (b) are equivalent. Also (b) implies (c) : Given $G \in$ (S) there is a neighborhood $V$ of $\pi(\mathbb{S})=\pi(\mathfrak{F})$ such that $G=V \cap B \supset V \cap A \in \mathfrak{F}$. Also (c) implies (b): If $\pi(\mathfrak{F}) \neq \pi(\mathbb{S})$, then $\pi(\mathfrak{F})$ and $\pi(\mathbb{S})$ have disjoint neighborhoods $V$ and $W$ in $E^{\prime \prime}$, and so $W \cap A$ is a set of $B$ containing no set of $\mathfrak{F}$.

Corollary. Let $A$ and $B \in \mathfrak{A}, \mathfrak{F} \in \beta_{A}$, and $\mathbb{S} \in \beta_{B}$. The following three conditions are equivalent:

(a) $\pi(\mathfrak{F})=\pi(\mathbb{S})$.

(b) There exists $C \in \mathfrak{U}$ such that $C \supset A \cup B$ and $g_{\sigma_{A}}(\widetilde{\jmath})=g_{\sigma_{B}}(\mathbb{S})$.

(c) There exists $C \in \mathfrak{A}$ and $\mathfrak{S} \in \beta_{0}$ such that $C \supset A \cup B$ and every set of $\mathfrak{S}$ contains a set of $\widetilde{F}$ and a set of $\mathbb{B}$.

Now let $R$ be the equivalence relation $\pi(\widetilde{F})=\pi(\$)$ on $\alpha, \eta$ the quotient space $\alpha / R, \rho$ the canonical mapping of $\alpha$ onto $\eta$, and $\sigma$ the mapping from $\eta$ into $E^{\prime \prime}$ such that $\pi=\sigma \rho$.

THEOREM 5. $\sigma$ is a homeomorphism of $\eta$ onto the $\mathscr{T}_{n}$-closure $\bar{E}$ of $E$ in $E^{\prime \prime}$.

Proof. We need only prove $\sigma(\eta)=\pi(\alpha) \supset \bar{E}$. Consider the dual system $\left\langle E^{\prime}, \bar{E}\right\rangle$. Since every $u \in E^{\prime}$ is uniformly continuous on $E$, the topology induced on $\bar{E}$ by $\mathscr{T}_{n}$ is admissible for this dual system. For $z \in \bar{E}$, there is a closed absolutely convex set $B \in \mathfrak{B}$ such that $|z u| \leqq 1$ for every $u \in B^{\circ}$. Hence, $z \in B^{\circ \circ}=$ the closure of $B$ in any admissible topology $=$ the $\mathscr{T}_{n}$-closure $\bar{B}$ of $B$.

For $B \in \mathfrak{A}$, the weakest topology on $\beta_{B}$ for which every function of the form $\widetilde{F} \rightarrow \mathfrak{F}(u)$ (for $u \in E^{\prime}$ ) is continuous will be called the weak topology of $\beta_{B}$. Clearly $\beta_{B}$ in the weak topology is homeomorphic 
with $\bar{B}$ in the topology induced on $\bar{B}$ by the weak-star topology of $E^{\prime \prime}$.

THEOREM 6. The following three conditions are equivalent:

(a) $\bar{E}=E^{\prime \prime}$;

(b) $\bar{B}$ is weak-star compact for every $B \in \mathfrak{U}$;

(c) $\beta_{B}$ is weakly compact for every $B \in \mathfrak{A}$.

Proof. Clearly (b) and (c) are equivalent. Also (a) implies (b); by the Alaoglu-Bourbaki theorem, for $B \in \mathfrak{A}$, the weak-star closure of $B$ in $E^{\prime \prime}=\bar{E}$ is weak-star compact; but since $\mathscr{T}_{n}$ is an admissible topology for the dual systm $\left\langle E^{\prime}, \bar{E}\right\rangle$, this weak-star closure is $\bar{B}$. Finally (b) implies (a): regarding $\mathfrak{B}$ as a total class of bounded subsets of $\bar{E}$, by the Mackey-Arens theorem $\mathscr{T}_{\mathfrak{B}}^{-}$is an admissible topology for the dual system $\left\langle E^{\prime}, \bar{E}\right\rangle$, and so $E^{\prime \prime}=\bar{E}$.

THEOREM 7. For $B \in \mathfrak{A}, \beta_{B}$ is weakly compact if and only if for every maximal weakly-convex filter $\mathfrak{F}$ on $B$, there is a maximal $\mathscr{T}$ convex filter on $B$ which is stronger than $\mathfrak{F}$.

Proof. Let $\beta_{B}^{w}$ be the space of all maximal weakly convex filters on $B$ and $\pi_{B}^{w}$ the homeomorphism of $\beta_{B}^{w}$ into $E^{\prime \prime}$ with the weak-star topology. In general $B \subset \pi_{B}\left(\beta_{B}\right)=\bar{B} \subset$ weak-star closure of $B=\pi_{B}^{w}\left(\beta_{B}^{w}\right)$.

If $\beta_{B}$ is weakly compact, then $\pi_{B}^{w}\left(\beta_{B}^{w}\right)=\pi_{B}\left(\beta_{B}\right)=\bar{B}$. So, for $\mathfrak{₹} \in \beta_{B}^{w}$, $\pi_{B}^{w}(\mathfrak{F}) \in \bar{B}$ and hence $\mathfrak{B}_{B}\left(\pi_{B}^{w}(\mathfrak{F})\right) \in \beta_{B}$ is stronger than $\widetilde{F}$.

Conversely, let $\mathfrak{F} \in \beta_{B}^{w}$ and $\mathbb{S} \in \beta_{B}$ stronger than $\widetilde{F}$. Then $\pi_{B}^{w}(\mathfrak{F})=$ $\pi_{B}(\mathbb{S})$, and so $\pi_{B}^{w}\left(\beta_{B}^{w}\right) \subset \pi_{B}\left(\beta_{B}\right)$.

CoRollary. $\bar{E}=E^{\prime \prime}$ if and only if, for every $B \in \mathfrak{A}$ and every maximal weakly-convex filter $\mathfrak{F}$ on $B$, there is a $\mathscr{T}$-convex filter on $B$ stronger than $\mathfrak{F}$.

3. Acknowledgement. The author wished to express his gratitude to Professor Ky Fan for the help and encouragement given him in the work presented here.

UNIVERSITY OF CINCINNATI 


\section{PACIFIC JOURNAL OF MATHEMATICS}

\section{EDITORS}

\author{
H. Samelson \\ Stanford University \\ Stanford, California \\ R. M. Blumenthal \\ University of Washington \\ Seattle, Washington 98105
}

\author{
J. Dugundu \\ University of Southern California \\ Los Angeles, California 90007 \\ *Richard Arens \\ University of California \\ Los Angeles, California 90024
}

\section{ASSOCIATE EDITORS}
E. F. BeCKENBACH
B. H. NeUMANN
F. WOLF
K. YosidA

\section{SUPPORTING INSTITUTIONS}

\author{
UNIVERSITY OF BRITISH COLUMBIA \\ CALIFORNIA INSTITUTE OF TECHNOLOGY \\ UNIVERSITY OF CALIFORNIA \\ MONTANA STATE UNIVERSITY \\ UNIVERSITY OF NEVADA \\ NEW MEXICO STATE UNIVERSITY \\ OREGON STATE UNIVERSITY \\ UNIVERSITY OF OREGON \\ OSAKA UNIVERSITY \\ UNIVERSITY OF SOUTHERN CALIFORNIA
}

\author{
STANFORD UNIVERSITY \\ UNIVERSITY OF TOKYO \\ UNIVERSITY OF UTAH \\ WASHINGTON STATE UNIVERSITY \\ UNIVERSITY OF WASHINGTON \\ * * * * \\ AMERICAN MATHEMATICAL SOCIETY \\ CALIFORNIA RESEARCH CORPORATION \\ SPACE TECHNOLOGY LABORATORIES \\ NAVAL ORDNANCE TEST STATION
}

Mathematical papers intended for publication in the Pacific Journal of Mathematics should by typewritten (double spaced). The first paragraph or two must be capable of being used separately as a synopsis of the entire paper. It should not contain references to the bibliography. No separate author's resumé is required. Manuscripts may be sent to any one of the four editors. All other communications to the editors should be addressed to the managing editor, Richard Arens, at the University of California, Los Angeles, California 90024.

50 reprints per author of each article are furnished free of charge; additional copies may be obtained at cost in multiples of 50 .

The Pacific Journal of Mathematics is published quarterly, in March, June, September, and December. Effective with Volume 13 the price per volume (4 numbers) is $\$ 18.00$; single issues, $\$ 5.00$. Special price for current issues to individual faculty members of supporting institutions and to individual members of the American Mathematical Society: $\$ 8.00$ per volume; single issues $\$ 2.50$. Back numbers are available.

Subscriptions, orders for back numbers, and changes of address should be sent to Pacific Journal of Mathematics, 103 Highland Boulevard, Berkeley 8, California.

Printed at Kokusai Bunken Insatsusha (International Academic Printing Co., Ltd.), No. 6, 2-chome, Fujimi-cho, Chiyoda-ku, Tokyo, Japan.

PUBLISHED BY PACIFIC JOURNAL OF MATHEMATICS, A NON-PROFIT CORPORATION

The Supporting Institutions listed above contribute to the cost of publication of this Journal, but they are not owners or publishers and have no responsibility for its content or policies.

* Basil Gordon, Acting Managing Editor until February 1, 1966. 


\section{Pacific Journal of Mathematics}

\section{Vol. 15, No. $3 \quad$ November, 1965}

David R. Arterburn and Robert James Whitley, Projections in the space of

bounded linear operators .................................

Robert McCallum Blumenthal, Joram Lindenstrauss and Robert Ralph Phelps,

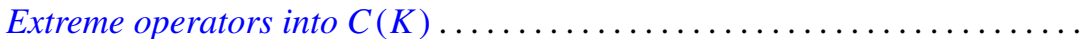

L. Carlitz, A note on multiple exponential sums ................... 757

Joseph A. Cima, A nonnormal Blaschke-quotient .................... 767

Paul Civin and Bertram Yood, Lie and Jordan structures in Banach algebras . . . 775

Luther Elic Claborn, Dedekind domains: Overrings and semi-prime

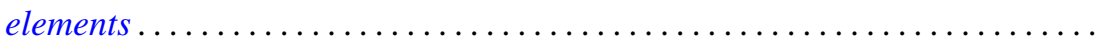

799

Luther Elic Claborn, Note generalizing a result of Samuel's .............. 805

George Bernard Dantzig, E. Eisenberg and Richard Warren Cottle, Symmetric

dual nonlinear programs ................................... 809

Philip J. Davis, Simple quadratures in the complex plane ............... 813

Edward Richard Fadell, On a coincidence theorem of F. B. Fuller ............ 825

Delbert Ray Fulkerson and Oliver Gross, Incidence matrices and interval

graphs ........................................ 835

Larry Charles Grove, Tensor products over $H^{*}$-algebras ..................

Deborah Tepper Haimo, $L^{2}$ expansions in terms of generalized heat polynomials

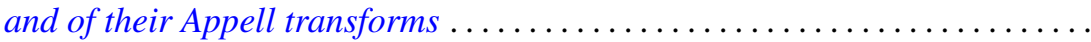

I. Martin (Irving) Isaacs and Donald Steven Passman, A chardcterization of groups in terms of the degrees of their characters ..........

Donald Gordon James, Integral invariants for vectors over local fields........ 905

Fred Krakowski, A remark on the lemma of Gauss ................... 917

Marvin David Marcus and H. Minc, A subdeterminant inequality ........... 921

Kevin Mor McCrimmon, Norms and noncommutative Jordan algebras ........ 925

Donald Earl Myers, Topologies for Laplace transform spaces ............... 957

Olav Njstad, On some classes of nearly open sets .................... 961

Milton Philip Olson, A characterization of conditional probability ........... 971

Barbara Osofsky, A counter-example to a lemma of Skornjakov .............. 985

Sidney Charles Port, Ratio limit theorems for Markov chains ............... 989

George A. Reid, A generalisation of $W^{*}$-algebras ...................... 1019

Robert Wells Ritchie, Classes of recursive functions based on Ackermann's

function ........................................... 1027

Thomas Lawrence Sherman, Properties of solutions of $n$th order linear

differential equations ........................................ 1045

Ernst Snapper, Inflation and deflation for all dimensions . .............. 1061

Kondagunta Sundaresan, On the strict and uniform convexity of certain Banach

spaces............................................. 1083

Frank J. Wagner, Maximal convex filters in a locally convex space .......... 1087

Joseph Albert Wolf, Translation-invariant function algebras on compact 